\title{
The behavior of depth functions of cover ideals of unimodular hypergraphs
}

\author{
Nguyen Thu Hang and Tran Nam Trung
}

\begin{abstract}
We prove that the cover ideals of all unimodular hypergraphs have the nonincreasing depth function property. Furthermore, we show that the index of depth stability of these ideals is bounded by the number of variables.
\end{abstract}

\section{Introduction}

Let $R=k\left[x_{1}, \ldots, x_{n}\right]$ be a polynomial ring over a given field $k$, and let $I$ be a homogeneous ideal in $R$. It is known by Brodmann [3] that $\operatorname{depth}\left(R / I^{s}\right)$ takes a constant value for large $s$. Moreover,

$$
\lim _{s \rightarrow \infty} \operatorname{depth} R / I^{s} \leqslant \operatorname{dim} R-\ell(I),
$$

where $\ell(I)$ is the analytic spread of $I$. The index of depth stability of $I$ is defined by

$$
\operatorname{dstab}(I):=\min \left\{s_{0} \geqslant 1 \mid \operatorname{depth} S / I^{s}=\operatorname{depth} S / I^{s_{0}} \text { for all } s \geqslant s_{0}\right\} .
$$

Two natural questions arise from Brodmann's theorem:

(1) What is the nature of the function $s \mapsto \operatorname{depth} R / I^{s}$ for $s \leqslant \operatorname{dstab}(I)$ ?

(2) What is a reasonable bound for $\operatorname{dstab}(I)$ ?

On the nature of the function $s \mapsto \operatorname{depth} R / I^{s}$ for $s \geqslant 1$, which is called the depth function of $I$, Herzog and Hibi [10] conjectured that the depth function of ideals can be any convergent nonnegative integer valued function. The answer is affirmative for bounded increasing functions (see [10]) and non-increasing functions (see [8]).

The behavior of depth functions, even for monomial ideals, is complicated (see e.g. [1]). Squarefree monomial ideals behave considerably better than monomial 
ideals in general, and Herzog and Hibi [10] asked whether depth functions of any squarefree monomial $I$ is non-increasing, that is

$$
\operatorname{depth}\left(R / I^{s}\right) \geqslant \operatorname{depth}\left(R / I^{s+1}\right) \text { for all } s \geqslant 1 \text {. }
$$

Kaiser, Stehlík and Škrekovski [18] gave a graph whose cover ideal is a counterexample to this question (see also [9] for more counterexamples).

It is an interesting problem to characterize squarefree monomial ideals $I$ with non-increasing depth function. This problem is difficult, and the characterization now has focused on certain families of monomial ideals, such as: ideals with all powers having a linear resolution (see [10]); cover ideals of bipartite graphs (see [6]); and ideals with constant depth function (see [8], [14] and [22]).

In this paper we prove that the cover ideal of a unimodular hypergraph has non-increasing depth function. Before stating our result we recall some terminology from graph theory (see [2] for more detail).

Let $\mathcal{V}=\{1, \ldots, n\}$, and let $\mathcal{E}$ be a family of distinct nonempty subsets of $\mathcal{V}$. The pair $\mathcal{H}=(\mathcal{V}, \mathcal{E})$ is called a hypergraph with vertex set $\mathcal{V}$ and edge set $\mathcal{E}$. Notice that a hypergraph generalizes the classical notion of a graph; a graph is a hypergraph for which every $E \in \mathcal{E}$ has cardinality two. One may also define a hypergraph by its incidence matrix $A(\mathcal{H})=\left(a_{i j}\right)$, with columns representing the edges $E_{1}, E_{2}, \ldots, E_{m}$ and rows representing the vertices $1,2, \ldots, n$ where $a_{i j}=0$ if $i \notin E_{j}$ and $a_{i j}=1$ if $i \in E_{j}$. A hypergraph $\mathcal{H}$ is said to be unimodular if its incident matrix is totally unimodular, i.e., every square submatrix of $A(\mathcal{H})$ has determinant equal to 0,1 or -1 .

A vertex cover of $\mathcal{H}$ is a subset of $\mathcal{V}$ which meets every edge of $\mathcal{H}$; a vertex cover is minimal if none of its proper subsets is itself a cover. For a subset $\tau=\left\{i_{1}, \ldots, i_{t}\right\}$ of $\mathcal{V}$, set $\mathbf{x}_{\tau}:=x_{i_{1}} \ldots x_{i_{t}}$. The cover ideal of $\mathcal{H}$ is then defined by:

$$
J(\mathcal{H}):=\left(\mathbf{x}_{\tau} \mid \tau \text { is a minimal vertex cover of } H\right) .
$$

It is well-known that there is one-to-one correspondence between squarefree monomial ideals of $R$ and cover ideals of hypergraphs on the vertex set $\mathcal{V}$.

The first main result of this paper is the following theorem.

Theorem 2.3. Let $\mathcal{H}$ be a unimodular hypergraph. Then, $J(\mathcal{H})$ has nonincreasing depth function.

We next address the question of when depth $R / I^{s}$ becomes stationary. Effective bounds of $\operatorname{dstab}(I)$ are known only for a few special classes of ideals $I$, such as complete intersection ideals (see [5]), squarefree Veronese ideals (see [10]), polymatroidal ideals (see [13]), edge ideals (see [25]). For any cover ideal of a unimodular hypergraph $\mathcal{H}$, we establish the universal and effective bound for $\operatorname{dstab}(J(\mathcal{H})$ ). Namely, 
Theorem 3.2. Let $\mathcal{H}=(\mathcal{V}, \mathcal{E})$ be a unimodular hypergraph with the vertex set $\mathcal{V}=\{1, \ldots, n\}$. Then,

$$
\operatorname{depth} R / J(\mathcal{H})^{s}=n-\ell(J(\mathcal{H})) \text { for all } s \geqslant n .
$$

In particular, $\operatorname{dstab}(J(\mathcal{H})) \leqslant n$.

When $\mathcal{H}$ is a bipartite graph $G$, this bound can be improved significantly by looking deeper into the structure of $G$. Let $\nu_{0}(G)$ be the ordered matching number of $G$ (see Definition 3.3). Then, we prove the following result.

Theorem 3.6. Let $G$ be a bipartite graph with $n$ vertices. Then,

$$
\operatorname{depth} R / J(G)^{s}=n-\nu_{0}(G)-1 \quad \text { for all } s \geqslant \nu_{0}(G) \text {. }
$$

In particular, $\operatorname{dstab}(J(G)) \leqslant \nu_{0}(G)$.

Moreover, this bound is sharp (see Proposition 3.7). It is worth mentioning that Herzog and Qureshi in [13] conjectured that dstab $(I)<\ell(I)$ for any squarefree monomial ideal $I$. Note that $\nu_{0}(G)=\ell(J(G))-1$ by [7, Corollary 2.9], so $\operatorname{dstab}(J(G))<\ell(J(G))$ by Theorem 3.6. Thus, the conjecture holds for $J(G)$.

Our approach is based on a generalized Hochster's formula for computing local cohomology modules of arbitrary monomial ideals formulated by Takayama [24]. Using this formula we are able to investigate the depth of powers of monomial ideals via the integer solutions of certain systems of linear inequalities. This allows us to use the theory of polytopes as the key role in this paper (see e.g. [15] and [16] for this approach).

The paper is organized as follows. In Section 1, we set up some basic notation and terminology for simplicial complex, the relationship between simplicial complexes and cover ideals of hypergraphs; and a generalization of Hochster's formula for computing local cohomology modules. In Section 2, we prove the non-increasing property for cover ideals of unimodular hypergraphs. In Section 3, we establish an upper bound for $\operatorname{dstab}(J(\mathcal{H}))$ of any unimodular hypergraph $\mathcal{H}$.

\section{Preliminary}

We first recall a relationship between cover ideals of hypergraphs and simplicial complexes. A simplicial complex on $\mathcal{V}=\{1, \ldots, n\}$ is a collection of subsets of $\mathcal{V}$ such that if $\sigma \in \Delta$ and $\tau \subseteq \sigma$ then $\tau \in \Delta$.

Definition 1.1. The Stanley-Reisner ideal associated to a simplicial complex $\Delta$ is the squarefree monomial ideal

$$
I_{\Delta}:=\left(x_{\tau} \mid \tau \notin \Delta\right) \subseteq R .
$$


Note that if $I$ is a squarefree monomial ideal, then it is a Stanley-Reisner ideal of the simplicial complex $\Delta(I):=\left\{\tau \subseteq \mathcal{V} \mid \mathbf{x}_{\tau} \notin I\right\}$. If $I$ is a monomial ideal (maybe not squarefree) we also use $\Delta(I)$ to denote the simplicial complex corresponding to the squarefree monomial ideal $\sqrt{I}$.

Let $\mathcal{F}(\Delta)$ be the set of facets of $\Delta$. If $\mathcal{F}(\Delta)=\left\{F_{1}, \ldots, F_{m}\right\}$, we write $\Delta=$ $\left\langle F_{1}, \ldots, F_{m}\right\rangle$. Then, $I_{\Delta}$ has the primary-decomposition (see [19, Theorem 1.7]):

$$
I_{\Delta}=\bigcap_{F \in \mathcal{F}(\Delta)}\left(x_{i} \mid i \notin F\right) .
$$

For $n \geqslant 1$, the $n$-th symbolic power of $I_{\Delta}$ is

$$
I_{\Delta}^{(n)}=\bigcap_{F \in \mathcal{F}(\Delta)}\left(x_{i} \mid i \notin F\right)^{n} .
$$

Let $\mathcal{H}=(\mathcal{V}, \mathcal{E})$ be a hypergraph. Then, the cover ideal of $\mathcal{H}$ can be written as

$$
J(\mathcal{H})=\bigcap_{E \in \mathcal{E}}\left(x_{i} \mid i \in E\right) .
$$

By this formula, when consider $J(\mathcal{H})$ without loss of generality we may assume that $\mathcal{H}$ is simple, i.e., whenever $E_{i}, E_{j} \in \mathcal{E}$ and $E_{i} \subseteq E_{j}$, then $E_{i}=E_{j}$. In this case, $J(\mathcal{H})$ is a Stanley-Reisner ideal with

$$
\Delta(J(\mathcal{H}))=\langle\mathcal{V} \backslash E \mid E \in \mathcal{E}\rangle
$$

Let $\mathfrak{m}:=\left(x_{1}, \ldots, x_{n}\right)$ be the maximal homogeneous ideal of $R$ and $I$ a monomial ideal in $R$. We can represent $\operatorname{depth} R / I$ via its local cohomology modules by

$$
\operatorname{depth}(R / I)=\min \left\{i \mid H_{\mathfrak{m}}^{i}(R / I) \neq 0\right\} .
$$

Since $R / I$ is an $\mathbb{N}^{n}$-graded algebra, $H_{\mathfrak{m}}^{i}(R / I)$ is a $\mathbb{Z}^{n}$-graded module over $R / I$ for every $i$. For each degree $\boldsymbol{\alpha}=\left(\alpha_{1}, \ldots, \alpha_{n}\right) \in \mathbb{Z}^{n}$, in order to compute $\operatorname{dim}_{k} H_{\mathfrak{m}}^{i}(R / I)_{\boldsymbol{\alpha}}$ we use a formula given by Takayama [24, Theorem 2.2] which is a generalization of Hochster's formula for the case $I$ is squarefree [17, Theorem 4.1].

Set $G_{\boldsymbol{\alpha}}:=\left\{i \mid \alpha_{i}<0\right\}$. For a subset $F \subseteq \mathcal{V}$, we let $R_{F}:=R\left[x_{i}^{-1} \mid i \in F \cup G_{\boldsymbol{\alpha}}\right]$. Define the simplicial complex $\Delta_{\alpha}(I)$ by

$$
\Delta_{\alpha}(I):=\left\{F \subseteq \mathcal{V} \backslash G_{\boldsymbol{\alpha}} \mid x^{\alpha} \notin I R_{F}\right\} .
$$

Lemma 1.2. ([24, Theorem 2.2]) $\operatorname{dim}_{k} H_{\mathfrak{m}}^{i}(R / I)_{\boldsymbol{\alpha}}=\operatorname{dim}_{k} \widetilde{H}_{i-\left|G_{\alpha}\right|-1}\left(\Delta_{\boldsymbol{\alpha}}(I) ; k\right)$.

If $\mathcal{H}$ is unimodular, the cover ideal $J(\mathcal{H})$ is normally torsion-free, i.e. $J(\mathcal{H})^{(s)}=$ $J(\mathcal{H})^{s}$ for all $s \geqslant 1$, by [12, Theorem 1.1]. Combining with [20, Lemma 1.3] we obtain: 
Lemma 1.3. Let $\mathcal{H}=(\mathcal{V}, \mathcal{E})$ be a unimodular hypergraph with $\mathcal{V}=\{1, \ldots, n\}$, and $\boldsymbol{\alpha}=\left(\alpha_{1}, \ldots, \alpha_{n}\right) \in \mathbb{N}^{n}$. Then, for every $s \geqslant 1$ we have

$$
\left.\Delta_{\boldsymbol{\alpha}}\left(J(\mathcal{H})^{s}\right)=\langle\mathcal{V} \backslash E| E \in \mathcal{E} \text { and } \sum_{i \in E} \alpha_{i} \leqslant s-1\right\rangle .
$$

For $F \subseteq \mathcal{V}$, set $S:=k\left[x_{i} \mid i \notin F\right]$ and $J^{\prime}:=J(\mathcal{H}) R_{F} \cap S$. Let $\mathcal{H}^{\prime}$ be a hypergraph on the vertex set $\mathcal{V}^{\prime}:=\mathcal{V} \backslash F$ with the edge set $\mathcal{E}^{\prime}=\{E \in \mathcal{E} \mid E \cap F=\varnothing\}$. By (1) we obtain:

$$
J(\mathcal{H}) R_{F} \cap S=J\left(\mathcal{H}^{\prime}\right) .
$$

In the sequel we also need the following two lemmas that will enable us to do induction on the number of variables.

Lemma 1.4. ([15, Lemma 1.3]) Let $I$ be a monomial ideal of $R$ and $F \subseteq\{1$, $\ldots, n\}$ such that $I R_{F} \neq R_{F}$. Let $S:=k\left[x_{i} \mid i \notin F\right]$ and $J:=I R_{F} \cap S$. Then, $\operatorname{depth} R / I \leqslant$ $|F|+\operatorname{depth} S / J$.

Lemma 1.5. ([15, Lemma 1.4]) Let $I$ be a monomial ideal of $R$ with $\operatorname{depth} R / I=d$. Assume that $H_{\mathfrak{m}}^{d}(R / I)_{\boldsymbol{\alpha}} \neq \mathbf{0}$ for some $\alpha=\left(\alpha_{1}, \ldots, \alpha_{n}\right) \in \mathbb{Z}^{n}$. Let $F:=$ $G_{\boldsymbol{\alpha}}, S:=k\left[x_{i} \mid i \notin F\right]$ and $J:=I R_{F} \cap S$. Then, $\operatorname{depth} R / I=\operatorname{depth} S / J+|F|$.

\section{The non-increasing property of depth functions}

In this section we prove the non-increasing property of cover ideals of unimodular hypergraphs. Throughout this section, let $\mathbf{e}_{1}, \ldots, \mathbf{e}_{n}$ be the canonical basis of $\mathbb{R}^{n}$.

Let $\mathcal{H}=(\mathcal{V}, \mathcal{E})$ be a unimodular hypergraph on the vertex set $\mathcal{V}=\{1, \ldots, n\}$. Suppose that $\mathcal{E}=\left\{E_{1}, \ldots, E_{m}\right\}$. Then, by (2)

$$
\Delta(J(\mathcal{H}))=\left\langle\mathcal{V} \backslash E_{1}, \ldots, \mathcal{V} \backslash E_{m}\right\rangle
$$

Now let $p \geqslant 0$ and $\boldsymbol{\alpha}=\left(\alpha_{1}, \ldots, \alpha_{n}\right) \in \mathbb{N}^{n}$ such that $H_{\mathfrak{m}}^{p}\left(R / J(\mathcal{H})^{s}\right)_{\boldsymbol{\alpha}} \neq \mathbf{0}$. By Lemma 1.2 we have

$$
\operatorname{dim}_{k} \widetilde{H}_{p-1}\left(\Delta_{\boldsymbol{\alpha}}\left(J(\mathcal{H})^{s}\right) ; k\right)=\operatorname{dim}_{k} H_{\mathfrak{m}}^{p}\left(R / J(\mathcal{H})^{s}\right)_{\boldsymbol{\alpha}}
$$

so that $\widetilde{H}_{p-1}\left(\Delta_{\boldsymbol{\alpha}}\left(J(\mathcal{H})^{s}\right) ; k\right) \neq \mathbf{0}$. In particular, $\Delta_{\boldsymbol{\alpha}}\left(J(\mathcal{H})^{s}\right) \neq \varnothing$. Thus, by Lemma 1.3 we may assume that $\Delta_{\boldsymbol{\alpha}}\left(J(\mathcal{H})^{s}\right)=\left\langle\mathcal{V} \backslash E_{1}, \ldots, \mathcal{V} \backslash E_{r}\right\rangle$ where $1 \leqslant r \leqslant m$. 
For each $t \geqslant 1$, let $C_{t} \subset \mathbb{R}^{n}$ to be the solution of the following system of linear inequalities:

$$
\begin{cases}\sum_{i \in E_{j}} x_{i}<t & \text { for } j=1, \ldots, r, \\ \sum_{i \in E_{j}} x_{i} \geqslant t & \text { for } j=r+1, \ldots, m, \\ x_{1} \geqslant 0, \ldots, x_{n} \geqslant 0 . & \end{cases}
$$

Then $C_{t}=t C_{1}$. Since $\boldsymbol{\alpha} \in C_{s}$, we have $(1 / s) \boldsymbol{\alpha} \in C_{1}$. Hence, $C_{1} \neq \varnothing$, and hence $C_{t} \neq \varnothing$. Moreover, by Lemma 1.3 one has

$$
\Delta_{\boldsymbol{\beta}}\left(J(\mathcal{H})^{t}\right)=\left\langle\mathcal{V} \backslash E_{1}, \ldots, \mathcal{V} \backslash E_{r}\right\rangle=\Delta_{\boldsymbol{\alpha}}\left(J(\mathcal{H})^{s}\right) \quad \text { for any } \boldsymbol{\beta} \in C_{t} \cap \mathbb{N}^{r}
$$

Let $\bar{C}_{t}$ be the closure of $C_{t}$ in $\mathbb{R}^{n}$ with respect to the Euclidean topology. Then, $\bar{C}_{t}=t \bar{C}_{1}$ and $\bar{C}_{1} \neq \varnothing$. Moreover, $\bar{C}_{t}$ is solutions in $\mathbb{R}^{n}$ of the following system:

$$
\begin{cases}\sum_{i \in E_{j}} x_{i} \leqslant t & \text { for } j=1, \ldots, r, \\ \sum_{i \in E_{j}} x_{i} \geqslant t & \text { for } j=r+1, \ldots, m, \\ x_{1} \geqslant 0, \ldots, x_{n} \geqslant 0 . & \end{cases}
$$

In particular, $\bar{C}_{t}$ is a convex polyhedron in $\mathbb{R}^{n}$.

Lemma 2.1. $\bar{C}_{1}$ is a polytope and $\operatorname{dim} \bar{C}_{1}=n$.

Proof. First we prove $C_{1}$ is bounded in $\mathbb{R}^{n}$. Indeed, let $\mathbf{y}=\left(y_{1}, \ldots, y_{n}\right) \in C_{1}$. For every $i=1, \ldots, n$, since $\Delta_{\boldsymbol{\alpha}}\left(J(\mathcal{H})^{s}\right)=\left\langle\mathcal{V} \backslash E_{1}, \ldots, \mathcal{V} \backslash E_{r}\right\rangle$ is not acyclic, it is not a cone over $i$, hence $i \in E_{j}$ for some $j=1, \ldots, r$. Together with the system (5) we get

$$
0 \leqslant y_{i} \leqslant \sum_{q \in E_{j}} y_{q}<1,
$$

so $C_{1}$ is bounded, as claimed.

Therefore, $\bar{C}_{1}$ is bounded too, and therefore $\bar{C}_{1}$ is a polytope in $\mathbb{R}^{n}$. Now we prove $\operatorname{dim} \bar{C}_{1}=n$. Since $C_{1} \neq \varnothing$, we can take a point $\beta=\left(\beta_{1}, \ldots, \beta_{n}\right) \in C_{1}$. From the system (5), there exists a real number $\varepsilon>0$ such that for all real numbers $\varepsilon_{1}, \ldots, \varepsilon_{n}$ with $0 \leqslant \varepsilon_{1}, \ldots, \varepsilon_{n} \leqslant \varepsilon$, we have $\beta+\varepsilon_{1} \mathbf{e}_{1}+\ldots+\varepsilon_{n} \mathbf{e}_{n} \in C_{1}$, so $\left[\beta_{1}, \beta_{1}+\varepsilon\right] \times \ldots \times\left[\beta_{n}, \beta_{n}+\right.$ $\varepsilon] \subseteq C_{1} \subseteq \bar{C}_{1}$. Thus, the polytope $\bar{C}_{1}$ is full dimensional in $\mathbb{R}^{n}$, as required.

We call a point of $\mathbb{R}^{n}$ is an integer point if all its coordinates are integers.

Lemma 2.2. Every vertex of $\bar{C}_{1}$ is an integer point. 
Proof. Let $\boldsymbol{\beta}=\left(\beta_{1}, \ldots, \beta_{n}\right) \in \mathbb{R}^{n}$ be a vertex of $\bar{C}_{1}$. Note that $\bar{C}_{1}$ is a polytope of full dimensional in $\mathbb{R}^{n}$. Moreover, $\bar{C}_{1}$ is the solutions in $\mathbb{R}^{n}$ of the system (6) with $t=1$. By [23, Formula 23 in p. 104], one has $\boldsymbol{\beta}$ must be the unique solution of a system of linear equations of the form

$$
\begin{cases}\sum_{i \in E_{j}} x_{i}=1 & \text { for } j \in S_{1} \subseteq\{1, \ldots, m\}, \\ x_{j}=0 & \text { for } j \in S_{2} \subseteq\{1, \ldots, n\}\end{cases}
$$

where $\left|S_{1}\right|+\left|S_{2}\right|=n$. By Cramer's rule, we can represent coordinates of $\boldsymbol{\beta}$ as

$$
\beta_{i}=\frac{D_{i}}{D} \quad \text { for } i=1, \ldots n,
$$

where $D, D_{1}, \ldots, D_{n} \in \mathbb{Z}$ and $D$ is the determinant of the matrix of this system.

Since the matrix of the system:

$$
\sum_{i \in E_{j}} x_{i}=1, \quad j \in S_{1}
$$

is a submatrix of $A(\mathcal{H})^{T}$, the transpose of $A(\mathcal{H})$. Thus, it is totally unimodular, and thus the matrix of the system $(7)$ is totally unimodular. This yields either $D=-1$ or $D=1$. Consequently, $\beta_{1}, \ldots, \beta_{n} \in \mathbb{N}^{n}$, i.e. $\boldsymbol{\beta}$ is an integer point.

We are now ready to prove the main result of this section.

Theorem 2.3. Let $\mathcal{H}$ be a unimodular hypergraph. Then, J(H) has nonincreasing depth function.

Proof. Fix any $s \geqslant 1$. We prove by induction on $n=|\mathcal{V}|$ that

$$
\operatorname{depth} R / J(\mathcal{H})^{s} \geqslant \operatorname{depth} R / J(\mathcal{H})^{s+1} \text {. }
$$

If $n \leqslant 2$, then $\operatorname{depth} R / J(\mathcal{H})^{s}=\operatorname{depth} R / J(\mathcal{H})^{s+1}$, so the theorem holds.

Assume that $n \geqslant 3$. Let $d:=\operatorname{depth} R / J(\mathcal{H})^{s}$. Then, $H_{\mathfrak{m}}^{d}\left(R / J(\mathcal{H})^{s}\right)_{\boldsymbol{\alpha}} \neq \mathbf{0}$ for some $\boldsymbol{\alpha} \in \mathbb{Z}^{n}$. Let $F:=G_{\boldsymbol{\alpha}}$. We now consider two cases:

Case 1. $(F \neq \varnothing)$ Let $\mathcal{V}^{\prime}:=\mathcal{V} \backslash F$ and $S:=k\left[x_{i} \mid i \in \mathcal{V}^{\prime}\right]$. Let $\mathcal{H}^{\prime}$ be the hypergraph on the vertex set $\mathcal{V}^{\prime}$ with the edge set $\mathcal{E}^{\prime}=\{E \in \mathcal{E} \mid E \cap F=\varnothing\}$. Then, by (4) we have $J\left(\mathcal{H}^{\prime}\right)=J(\mathcal{H}) R_{F} \cap S$. Since $\mathcal{H}$ is unimodular, $\mathcal{H}^{\prime}$ is unimodular.

By Lemma 1.5 we have $\operatorname{depth} R / J(\mathcal{H})^{s}=\operatorname{depth} S / J\left(\mathcal{H}^{\prime}\right)^{s}+|F|$. By Lemma 1.4 we have $\operatorname{depth} R / J(\mathcal{H})^{s+1} \leqslant \operatorname{depth} S / J\left(\mathcal{H}^{\prime}\right)^{s+1}+|F|$.

On the other hand, since $\left|\mathcal{V}^{\prime}\right|=|\mathcal{V}|-|F|<|\mathcal{V}|$, by the induction hypothesis we have $\operatorname{depth} S / J\left(\mathcal{H}^{\prime}\right)^{s} \geqslant \operatorname{depth} S / J\left(\mathcal{H}^{\prime}\right)^{s+1}$. It follows that

$$
\begin{aligned}
\operatorname{depth} R / J(\mathcal{H})^{s+1} & \leqslant \operatorname{depth} S / J\left(\mathcal{H}^{\prime}\right)^{s+1}+|F| \leqslant \operatorname{depth} S / J\left(\mathcal{H}^{\prime}\right)^{s}+|F| \\
& =\operatorname{depth} R / J(\mathcal{H})^{s} .
\end{aligned}
$$


Case 2. $\left(F=\varnothing\right.$, i.e. $\left.\boldsymbol{\alpha}=\left(\alpha_{1}, \ldots, \alpha_{n}\right) \in \mathbb{N}^{n}\right)$ By Lemma 1.2 we have

$$
\operatorname{dim}_{k} \widetilde{H}_{d-1}\left(\Delta_{\boldsymbol{\alpha}}\left(I(\mathcal{H})^{s}\right) ; k\right)=\operatorname{dim}_{k} H_{\mathfrak{m}}^{d}\left(R / J(\mathcal{H})^{s}\right)_{\boldsymbol{\alpha}},
$$

so $\widetilde{H}_{d-1}\left(\Delta_{\boldsymbol{\alpha}}\left(I(\mathcal{H})^{s}\right) ; k\right) \neq \mathbf{0}$.

Suppose that $\mathcal{E}=\left\{E_{1}, \ldots, E_{m}\right\}$. By (2) and Lemma 1.2 we may assume that $\mathcal{F}\left(\Delta_{\boldsymbol{\alpha}}\left(J(\mathcal{H})^{s}\right)=\left\{\mathcal{V} \backslash E_{1}, \ldots, \mathcal{V} \backslash E_{r}\right\}\right.$ for some $1 \leqslant r \leqslant m$.

By Lemma 1.3 we have

$$
\begin{cases}\sum_{i \in E_{j}} \alpha_{i}<s & \text { for } j=1, \ldots, r, \\ \sum_{i \in E_{j}} \alpha_{i} \geqslant s & \text { for } j=r+1, \ldots, m .\end{cases}
$$

On the other hand, by Lemmas 2.1 and 2.2, the following system of linear inequalities

$$
\begin{cases}\sum_{i \in E_{j}} x_{i} \leqslant 1 & \text { for } j=1, \ldots, r, \\ \sum_{i \in E_{j}} x_{i} \geqslant 1 & \text { for } j=r+1, \ldots, m, \\ x_{1} \geqslant 0, \ldots, x_{n} \geqslant 0, & \end{cases}
$$

has at least one integer solution, say $\boldsymbol{\beta}=\left(\beta_{1}, \ldots, \beta_{n}\right) \in \mathbb{N}^{n}$.

Let $\boldsymbol{\gamma}:=\boldsymbol{\alpha}+\boldsymbol{\beta}$ so that $\boldsymbol{\gamma} \in \mathbb{N}^{n}$. Write $\boldsymbol{\gamma}=\left(\gamma_{1}, \ldots, \gamma_{n}\right)$. From (9) and (10) we yields

$$
\begin{cases}\sum_{i \in E_{j}} \gamma_{i}<s+1 & \text { for } j=1, \ldots, r, \\ \sum_{i \in E_{j}} \gamma_{i} \geqslant s+1 & \text { for } j=r+1, \ldots, m .\end{cases}
$$

Together with Lemma 1.3 we have $\Delta_{\boldsymbol{\gamma}}\left(J(\mathcal{H})^{s+1}\right)=\Delta_{\boldsymbol{\alpha}}\left(J(\mathcal{H})^{s}\right)$.

Since $\widetilde{H}_{d-1}\left(\Delta_{\boldsymbol{\alpha}}\left(J(\mathcal{H})^{s}\right) ; k\right) \neq 0$, we have $\widetilde{H}_{d-1}\left(\Delta_{\boldsymbol{\gamma}}\left(J(\mathcal{H})^{s+1}\right) ; k\right) \neq 0$. Together with Lemma 1.2 we get $H_{\mathfrak{m}}^{d}\left(R / J(\mathcal{H})^{s+1}\right)_{\boldsymbol{\gamma}} \neq \mathbf{0}$, so $H_{\mathfrak{m}}^{d}\left(R / J(\mathcal{H})^{s+1}\right) \neq \mathbf{0}$. This implies $\operatorname{depth} R / J(\mathcal{H})^{s+1} \leqslant d=\operatorname{depth} R / J(\mathcal{H})^{s}$. The proof is complete.

In the case $\mathcal{H}$ is a bipartite graph. Then, it is unimodular by [2, Theorem 5]. As a consequence of Theorem 2.3 we recover [6, Theorem 3.2].

Corollary 2.4. $J(G)$ has non-increasing depth function for any bipartite graph $G$.

\section{The index of depth stability}

In this section we establish the upper bound of $\operatorname{dstab}(J(\mathcal{H}))$ for the cover ideal of any unimodular hypergraph $\mathcal{H}$. Recall that the analytic spread of a homogeneous 
ideal $I$ of $R$ is defined by

$$
\ell(I):=\operatorname{dim} \mathcal{R}(I) / \mathfrak{m} \mathcal{R}(I),
$$

where $\mathcal{R}(I)=\bigoplus_{s=0}^{\infty} I^{s}$ is the Rees ring of $I$.

Lemma 3.1. Let $\mathcal{H}$ be a unimodular hypergraph. Then,

$$
\lim _{s \rightarrow \infty} \operatorname{depth} R / J(\mathcal{H})^{s}=\operatorname{dim} R-\ell(J(\mathcal{H})) .
$$

Proof. Since $\mathcal{H}$ is unimodular, $J(\mathcal{H})$ is totally torsion-free by [12, Theorem 1.1]. The lemma now follows from [11, Proposition 10.3.2 and Theorem 10.3.13].

We are in position to prove the main result of this section.

Theorem 3.2. Let $\mathcal{H}=(\mathcal{V}, \mathcal{E})$ be a unimodular hypergraph with the vertex set $\mathcal{V}=\{1, \ldots, n\}$. Then,

$$
\operatorname{depth} R / J(\mathcal{H})^{s}=n-\ell(J(\mathcal{H})) \text { for all } s \geqslant n .
$$

In particular, $\operatorname{dstab}(J(\mathcal{H})) \leqslant n$.

Proof. Since $H$ is unimodular, by Theorem 2.3 and Lemma 3.1 we have

$$
\operatorname{dstab}(J(\mathcal{H}))=\min \left\{s \geqslant 1 \mid \operatorname{depth} R / J(\mathcal{H})^{s}=\operatorname{dim} R-\ell(J(\mathcal{H})\} .\right.
$$

Thus, it remains to show that $\operatorname{dstab}(J(\mathcal{H})) \leqslant n$.

We prove the assertion by induction on $n$. If $n \leqslant 2$, then $\operatorname{depth} R / J(\mathcal{H})^{s}=$ $\operatorname{depth} R / J(\mathcal{H})$ for all $s \geqslant 1$, and then the assertion holds.

Assume that $n \geqslant 3$. Let $s:=\operatorname{dstab}(J(\mathcal{H}))$ and $d:=n-\ell(J(\mathcal{H}))$. Then, $H_{\mathfrak{m}}^{d}(R /$ $\left.J(\mathcal{H})^{s}\right)_{\boldsymbol{\alpha}} \neq \mathbf{0}$ for some $\boldsymbol{\alpha} \in \mathbb{Z}^{n}$. Let $F:=G_{\boldsymbol{\alpha}}$. We now consider two cases:

Case 1. $(F \neq \varnothing)$ Let $\mathcal{V}^{\prime}:=\mathcal{V} \backslash F$ and $S:=k\left[x_{i} \mid i \in \mathcal{V}^{\prime}\right]$. Let $\mathcal{H}^{\prime}$ be the hypergraph on the vertex set $\mathcal{V}^{\prime}$ with the edge set $\mathcal{E}^{\prime}=\{E \in \mathcal{E} \mid E \cap F=\varnothing\}$. Then, by (4) we have $J\left(\mathcal{H}^{\prime}\right)=J(\mathcal{H}) R_{F} \cap S$. Since $\mathcal{H}$ is unimodular, $\mathcal{H}^{\prime}$ is unimodular too.

Note that $\operatorname{depth} R / J(\mathcal{H})^{s}=\operatorname{depth} S / J\left(\mathcal{H}^{\prime}\right)^{s}+|F|$ by Lemma 1.5. Now let $p:=$ $\operatorname{dstab}\left(J\left(\mathcal{H}^{\prime}\right)\right)$. Together with Lemma 1.4 and Theorem 2.3 we have

$$
\operatorname{depth} S / J\left(\mathcal{H}^{\prime}\right)^{p} \geqslant \operatorname{depth} R / J(\mathcal{H})^{p}-|F| \geqslant \operatorname{depth} R / J(\mathcal{H})^{s}-|F|=\operatorname{depth} S / J\left(\mathcal{H}^{\prime}\right)^{s} .
$$

Together with Theorem 2.3, this fact follows that $\operatorname{depth} S / J\left(\mathcal{H}^{\prime}\right)^{s}=\operatorname{depth} S / J\left(\mathcal{H}^{\prime}\right)^{p}$. Hence, the inequalities above yields $\operatorname{depth} R / J(H)^{p}=\operatorname{depth} R / J(\mathcal{H})^{s}$. By combining this with (11), we get $s \leqslant p$.

On the other hand, $p \leqslant\left|\mathcal{V}^{\prime}\right|<|\mathcal{V}|$ by the induction hypothesis. Thus, $s<n$, and the assertion holds for this case. 
Case 2. $\left(F=\varnothing\right.$, i.e. $\left.\boldsymbol{\alpha}=\left(\alpha_{1}, \ldots, \alpha_{n}\right) \in \mathbb{N}^{n}\right)$ By Lemma 1.2 we have

$$
\operatorname{dim}_{k} \widetilde{H}_{d-1}\left(\Delta_{\boldsymbol{\alpha}}\left(I(\mathcal{H})^{s}\right) ; k\right)=\operatorname{dim}_{k} H_{\mathfrak{m}}^{d}\left(R / J(\mathcal{H})^{s}\right)_{\boldsymbol{\alpha}},
$$

so $\widetilde{H}_{d-1}\left(\Delta_{\boldsymbol{\alpha}}\left(I(\mathcal{H})^{s}\right) ; k\right) \neq \mathbf{0}$.

Suppose that $\mathcal{E}=\left\{E_{1}, \ldots, E_{m}\right\}$. By (2) and Lemma 1.2 we may assume that $\mathcal{F}\left(\Delta_{\boldsymbol{\alpha}}\left(J(\mathcal{H})^{s}\right)\right)=\left\{\mathcal{V} \backslash E_{1}, \ldots, \mathcal{V} \backslash E_{r}\right\}$ for some $1 \leqslant r \leqslant m$.

For every $t \geqslant 1$, let $C_{t}$ the set of solutions in $\mathbb{R}^{n}$ of the following system of linear inequalities

$$
\begin{cases}\sum_{i \in E_{j}} x_{i}<t & \text { for } j=1, \ldots, r, \\ \sum_{i \in E_{j}} x_{i} \geqslant t & \text { for } j=r+1, \ldots, m, \\ x_{1} \geqslant 0, \ldots, x_{n} \geqslant 0 . & \end{cases}
$$

Let $\bar{C}_{t}$ be closure of $C_{t}$ in $\mathbb{R}^{n}$. Then, by (12), $\bar{C}_{t}$ is the set of solutions in $\mathbb{R}^{n}$ of the following system of linear inequalities

$$
\begin{cases}\sum_{i \in E_{j}} x_{i} \leqslant t & \text { for } j=1, \ldots, r, \\ \sum_{i \in E_{j}} x_{i} \geqslant t & \text { for } j=r+1, \ldots, m, \\ x_{1} \geqslant 0, \ldots, x_{n} \geqslant 0 . & \end{cases}
$$

By Lemmas 2.1 and 2.2, we conclude that $\bar{C}_{1}$ is a polytope of full dimensional in $\mathbb{R}^{n}$ with all integer vertices.

If $\bar{C}_{1}$ has no supporting hyperplanes of the form $\sum_{i \in E_{j}} x_{i}=1$ for all $j=r+$ $1, \ldots, m$. In this case, we imply that the zero vector $\boldsymbol{\beta}=(0, \ldots, 0) \in \bar{C}_{1}$, and so $\boldsymbol{\beta} \in$ $C_{1} \cap \mathbb{N}^{n}$.

If $\bar{C}_{1}$ has a supporting hyperplane of the form $\sum_{i \in E_{j}} x_{i}=1$ for some $j=r+$ $1, \ldots, m$. We may assume such a supporting hyperplane is $\sum_{i \in E_{m}} x_{i}=1$; and let $F$ be the facet of $\bar{C}_{1}$ determined by this hyperplane. Now take $n$ vertices of $\bar{C}_{1}$ lying in $F$, say $\boldsymbol{\alpha}^{1}, \ldots, \boldsymbol{\alpha}^{n}$, such that they are affinely independent. Let $\boldsymbol{\beta}:=\left(\boldsymbol{\alpha}^{1}+\ldots+\boldsymbol{\alpha}^{n}\right) / n \in$ $\bar{C}_{1}$. Then, $\boldsymbol{\beta}$ is a relative interior point of $F$, so that it does not belong to any another facet of $\bar{C}_{1}$. Thus, By (13), $\boldsymbol{\beta}$ satisfies the following system:

$$
\begin{cases}\sum_{i \in E_{j}} x_{i}<1 & \text { for } j=1, \ldots, r, \\ \sum_{i \in E_{j}} x_{i} \geqslant 1 & \text { for } j=r+1, \ldots, m, \\ x_{1} \geqslant 0, \ldots, x_{n} \geqslant 0 . & \end{cases}
$$

This forces $\boldsymbol{\beta} \in C_{1}$. Thus, $n \boldsymbol{\beta} \in C_{n} \cap \mathbb{N}^{n}$.

Let $\boldsymbol{\gamma}=n \boldsymbol{\beta} \in C_{n} \cap \mathbb{N}^{n}$. Then, by (14) and Lemma 1.3 we have $\Delta_{\boldsymbol{\gamma}}\left(J(\mathcal{H})^{n}\right)=$ $\Delta_{\boldsymbol{\alpha}}\left(J(\mathcal{H})^{s}\right)$, so $\widetilde{H}_{d-1}\left(\Delta_{\boldsymbol{\gamma}}\left(J(\mathcal{H})^{n}\right) ; k\right) \neq \mathbf{0}$. Together with Lemma 1.2 we deduce that 
$H_{\mathfrak{m}}^{d}\left(R / J(\mathcal{H})^{n}\right) \neq \mathbf{0} . \quad$ Consequently, $\operatorname{depth} R / J(\mathcal{H})^{n} \leqslant d . \quad$ Thus, $\operatorname{depth} R / J(\mathcal{H})^{n}=d$, and thus $s \leqslant n$ by (11), as required.

In the case $\mathcal{H}$ is a bipartite graph we will establish a better bound for $\operatorname{dstab}(J(\mathcal{H}))$. We first recall some terminology from the graph theory. Let $G=$ $(V(G), E(G))$ be a simple graph. Let $v(G)$ denote the number of vertices of $G$. A set of vertices $S \subseteq V(G)$ is called independent if $\{v, w\} \notin E(G)$ for any $v, w \in S$. A set $M \subseteq E(G)$ is a matching of $G$ if any two distinct edges of $M$ have no vertex in common. Let $M=\left\{\left\{a_{i}, b_{i}\right\} \mid i=1, \ldots, m\right\}$ be a nonempty matching of $G$. According to [7], we say that $M$ is an ordered matching if:

- $\left\{a_{1}, \ldots, a_{m}\right\}$ is a set of independent vertices,

- $\left\{a_{i}, b_{j}\right\} \in E(G)$ implies $i \leqslant j$.

Definition 3.3. The ordered matching number of $G$ is:

$$
\nu_{0}(G):=\max \{|M| \mid M \subseteq E(G) \text { is an ordered matching of } G\} .
$$

Assume that $G$ is bipartite with $V(G)=\{1, \ldots, n\}$. To describe $\Delta_{\boldsymbol{\alpha}}\left(J(G)^{s}\right)$ for $\boldsymbol{\alpha}=\left(\alpha_{1}, \ldots, \alpha_{n}\right) \in \mathbb{N}^{n}$ and $s \geqslant 1$, in more explicit way, for every edge $\{i, j\}$ of $G$ we let $F_{i j}:=\{1, \ldots, n\} \backslash\{i, j\}$. Then,

$$
\mathcal{F}(\Delta(J(G)))=\left\{F_{i j} \mid\{i, j\} \in E(G)\right\}
$$

and by Lemma 1.3 we obtain

$$
\left.\Delta_{\boldsymbol{\alpha}}\left(J(G)^{s}\right)=\left\langle F_{i j}\right| \alpha_{i}+\alpha_{j} \leqslant s-1 \text { and }\{i, j\} \in E(G)\right\rangle .
$$

Lemma 3.4. Let $G$ be a bipartite graph with bipartition $(X, Y)$. If $v(G)=$ $2 \nu_{0}(G)$, then $G$ has an ordered matching $M=\left\{\left\{a_{i}, b_{i}\right\} \mid i=1, \ldots, m\right\}$ where $m=\nu_{0}(G)$, such that

$$
X=\left\{a_{1}, \ldots, a_{m}\right\} \quad \text { and } \quad Y=\left\{b_{1}, \ldots, b_{m}\right\} .
$$

Proof. We prove by induction on $\nu_{0}(G)$. If $\nu_{0}(G)=1$, then $G$ is an edge, and then the lemma is obvious.

Assume that $\nu_{0}(G)=m \geqslant 2$. Let $\left\{\left\{x_{i}, y_{i}\right\} \mid i=1, \ldots, m\right\}$ be an ordered matching of $G$ such that

- $\left\{x_{1}, \ldots, x_{m}\right\}$ is an independent set of $G$;

- $\left\{x_{i}, y_{j}\right\} \in E(G)$ implies $i \leqslant j$.

This implies $\operatorname{deg}_{G}\left(x_{m}\right)=1$. Let $H:=G \backslash\left\{x_{m}, y_{m}\right\}$. Then, $H$ is a bipartite graph with so that $v(H)=v(G)-2=2(m-1)$ and $\nu_{0}(H)=m-1$. We now distinguish two cases: 
Case 1. $\left(x_{m} \in X\right)$ Then, $y_{m} \in Y$ and $H$ has a bipartition $\left(X \backslash\left\{x_{m}\right\}, Y \backslash\left\{y_{m}\right\}\right)$. Since $\nu_{0}(H)=m-1<m$, by the induction hypothesis we imply that $H$ has an ordered matching $\left\{a_{i} b_{i} \mid i=1, \ldots, m-1\right\}$ such that

$$
X \backslash\left\{x_{m}\right\}=\left\{a_{1}, \ldots, a_{m-1}\right\} \quad \text { and } \quad Y \backslash\left\{y_{m}\right\}=\left\{b_{1}, \ldots, b_{m-1}\right\} .
$$

Note that $\operatorname{deg}_{G}\left(x_{m}\right)=1, x_{m} \in X$ and $y_{m} \in Y$. Therefore, $\left\{a_{1} b_{1}, \ldots, a_{m-1} b_{m-1}, x_{m} y_{m}\right\}$ is a desired ordered matching.

Case 2. $\left(x_{m} \in Y\right)$ Then $y_{m} \in X$ and $H$ has a bipartition $\left(Y \backslash\left\{x_{m}\right\}, X \backslash\left\{y_{m}\right\}\right)$. Since $\nu_{0}(H)=m-1<m$, by the induction hypothesis we imply that $H$ has an ordered matching $\left\{a_{i} b_{i} \mid i=1, \ldots, m-1\right\}$ such that

$$
Y \backslash\left\{x_{m}\right\}=\left\{a_{1}, \ldots, a_{m-1}\right\} \quad \text { and } \quad X \backslash\left\{y_{m}\right\}=\left\{b_{1}, \ldots, b_{m-1}\right\} .
$$

Note that $\operatorname{deg}_{G}\left(x_{m}\right)=1, x_{m} \in X$ and $y_{m} \in Y$. Thus, $\left\{a_{1} b_{1}, \ldots, a_{m-1} b_{m-1}, x_{m} y_{m}\right\}$ is an ordered matching of $G$. This implies that

$$
\left\{y_{m} x_{m}, b_{m-1} a_{m-1}, \ldots, b_{1} a_{1}\right\}
$$

is a desired ordered matching, and the proof of the lemma is complete.

Lemma 3.5. ([15, Lemma 3.5]) Let $G$ be a graph with $2 m$ vertices. Then, depth $R / J(G)=m-1$ if and only if $G$ consists of $m$ disjoint edges.

If $G$ be a bipartite graph, then by Theorem 3.2 we have $\operatorname{dstab}(J(G)) \leqslant n$. This bound is refined as $\operatorname{dstab}(J(G)) \leqslant 2 \nu_{0}(G)-1$ by [15, Theorem 3.4]. By the same method we improve this bound as follows.

Theorem 3.6. Let $G$ be a bipartite graph with $n$ vertices. Then,

$$
\operatorname{depth} R / J(G)^{s}=n-\nu_{0}(G)-1 \quad \text { for all } s \geqslant \nu_{0}(G) \text {. }
$$

In particular, $\operatorname{dstab}(J(G)) \leqslant \nu_{0}(G)$.

Proof. Let $m:=\nu_{0}(G)$ and let $\left\{\left\{a_{i}, b_{i}\right\} \mid i=1, \ldots, m\right\}$ be an ordered matching of $G$. Let $H$ be the induced subgraph of $G$ on the vertex set $\left\{a_{i}, b_{i} \mid i=1, \ldots, m\right\}$. Then $H$ is a bipartite graph with $\nu_{0}(H)=m$ and $v(H)=2 \nu_{0}(H)$. By Lemma 3.4, $H$ has an ordered matching $\left\{\left\{x_{i}, y_{i}\right\} \mid i=1, \ldots, m\right\}$ such that $\left(\left\{x_{1}, \ldots, x_{m}\right\},\left\{y_{1}, \ldots, y_{m}\right\}\right)$ is a bipartition of $H$.

Without loss of generality we may assume that $x_{i}=i$ and $y_{i}=m+i$ for all $i=1, \ldots, m$. Let $F:=\{2 m+1, \ldots, n\}$ and $S:=k\left[x_{1}, \ldots, x_{2 m}\right]$.

We now define $\boldsymbol{\alpha}=\left(\alpha_{1}, \ldots, \alpha_{2 m}\right) \in \mathbb{N}^{2 m}$ as follows

$$
\alpha_{j}:= \begin{cases}j-1 & \text { for } j=1, \ldots, m, \\ 2 m-j & \text { for } j=m+1, \ldots, 2 m\end{cases}
$$


Since $H$ has an ordered matching $\{\{i, m+i\} \mid i=1, \ldots, m\}$ and a bipartition

$$
(\{1, \ldots, m\},\{m+1, \ldots, 2 m\}),
$$

we imply that

$$
\Delta_{\boldsymbol{\alpha}}\left(J(H)^{m}\right)=\left\langle F_{i, m+i} \mid i=1, \ldots, m\right\rangle .
$$

Let $\Lambda:=\Delta_{\boldsymbol{\alpha}}\left(J(H)^{m}\right)$. By Alexander duality (see [4, Lemma 5.5.3]) we have

$$
\operatorname{dim}_{k} \widetilde{H}_{m-2}(\Lambda ; k)=\operatorname{dim}_{k} \widetilde{H}_{m-1}\left(\Lambda^{*} ; k\right) .
$$

Since $\Lambda=\left\langle F_{i, m+i} \mid i=1, \ldots, m\right\rangle$, one has

$$
\Lambda^{*}=\langle\{1\},\{m+1\}\rangle *\langle\{2\},\{m+2\}\rangle * \ldots *\langle\{m\},\{2 m\}\rangle .
$$

Thus $\operatorname{dim}_{k} \widetilde{H}_{m-1}\left(\Lambda^{*} ; k\right) \neq 0$, and thus $\operatorname{dim}_{k} \widetilde{H}_{m-2}(\Lambda ; k) \neq 0$.

On the hand, by Lemma 1.2 we have

$$
\operatorname{dim}_{k} H_{\mathfrak{n}}^{m-1}\left(S / J(H)^{m}\right)_{\boldsymbol{\alpha}}=\operatorname{dim}_{k} \widetilde{H}_{m-2}(\Lambda ; k)
$$

where $\mathfrak{n}=\left(x_{1}, \ldots, x_{2 m}\right) \subset S$, hence $H_{\mathfrak{n}}^{m-1}\left(S / J(H)^{m}\right) \neq \mathbf{0}$, and hence

$$
\operatorname{depth} S / J(H)^{m} \leqslant m-1 \text {. }
$$

Let $F:=\{2 m+1, \ldots, n\}$. Then, $J(H)=J(G) R_{F} \cap S$ and $J(H)^{m}=J(G)^{m} R_{F} \cap S$. Together Inequality (16) and Lemma 1.4 we have

$\operatorname{depth} R / J(G)^{m} \leqslant|F|+\operatorname{depth} S / J(H)^{m} \leqslant(n-2 m)+(m-1)=n-\nu_{0}(G)-1$.

On the other hand, by [7, Corollary 2.9] we have $\ell(J(G))=\nu_{0}(G)+1$. Together with Theorems 2.3 and 3.2 , this fact gives

$$
\operatorname{depth} R / J(G)^{m} \geqslant n-\nu_{0}(G)-1 \text {. }
$$

Thus, depth $R / J(G)^{m}=n-\nu_{0}(G)-1$.

Finally, by Theorems 2.3 and 3.2 we have the sequence $\left\{\operatorname{depth} R / J(G)^{s}\right\}_{s} \geqslant 1$ is non-increasing and bounded below by $n-\nu_{0}(G)-1$, we obtain

$$
\operatorname{depth} R / J(G)^{s}=n-\nu_{0}(G)-1, \quad \text { for all } s \geqslant m=\nu_{0}(G),
$$

as required.

Finally, we construct an example to show that the bound in Theorem 3.6 is sharp. 
Proposition 3.7. Let $m \geqslant 1$. Let $G$ be a graph with the vertex set $V(G)=$ $\{1, \ldots, 2 m\}$ and the edge set

$$
E(G)=\{\{i, m+j\} \mid 1 \leqslant i \leqslant j \leqslant m\} .
$$

Then $\nu_{0}(G)=m$ and

$$
\operatorname{depth} R / J(G)^{s}=2 m-\nu_{0}(G)-1 \Longleftrightarrow s \geqslant \nu_{0}(G) \text {. }
$$

Proof. The equality $\nu_{0}(G)=m$ is clear. By Theorem 3.6 it suffices to show that if depth $R / J(G)^{s}=m-1$, then $s \geqslant m$.

Since depth $R / J(G)^{s}=m-1$, there is $\boldsymbol{\alpha}=\left(\alpha_{1}, \ldots, \alpha_{2 m}\right) \in \mathbb{Z}^{2 m}$ such that

$$
H_{\mathfrak{m}}^{m-1}\left(R / J(G)^{s}\right)_{\boldsymbol{\alpha}} \neq \mathbf{0}
$$

We first claim that $\boldsymbol{\alpha} \in \mathbb{N}^{2 m}$. Indeed, let $F:=G_{\boldsymbol{\alpha}}$ and $S:=k\left[x_{i} \mid i \notin F\right]$. Let $H$ be an induced subgraph of $G$ on the vertex set $[2 m] \backslash F$. Then, $J(H)=J(G) R_{F} \cap S$. By Lemma 1.5 we have

$$
\operatorname{depth} S / J(H)^{s}=\operatorname{depth} R / J(G)^{s}-|F|=m-1-|F|=\operatorname{dim} S-m-1 .
$$

Together with Theorems 2.3 and 3.2 we deduce that $\operatorname{dim} S-m-1 \geqslant \operatorname{dim} S-$ $\nu_{0}(H)-1$, and so $\nu_{0}(H) \geqslant m$. This inequality forces $\nu_{0}(H)=m$. In particular, $V(H)=V(G)$ and $G_{\boldsymbol{\alpha}}=\varnothing$. This yields $\boldsymbol{\alpha} \in \mathbb{N}^{2 m}$, as claimed.

Let $G^{\prime}$ be a subgraph of $G$ consists of all edges $\{i, j\}$ of $G$ such that $\alpha_{i}+\alpha_{j} \leqslant$ $s-1$. By Lemma 15 we have $\Delta_{\boldsymbol{\alpha}}\left(J(G)^{s}\right)=\left\langle F_{i j} \mid\{i, j\} \in E\left(G^{\prime}\right)\right\rangle$.

Since $\widetilde{H}_{m-2}\left(\Delta_{\boldsymbol{\alpha}}\left(J(G)^{s}\right) ; k\right) \neq \mathbf{0}$ by $(17)$ and Lemma 1.2 , we have $\Delta_{\boldsymbol{\alpha}}\left(J(G)^{s}\right)$ is not a cone, and so $V\left(G^{\prime}\right)=V(G)$.

Let $\boldsymbol{\beta}=(0,0, \ldots, 0) \in \mathbb{N}^{2 m}$. Then,

$$
\Delta_{\boldsymbol{\beta}}\left(J\left(G^{\prime}\right)\right)=\left\langle F_{i j} \mid\{i, j\} \in E\left(G^{\prime}\right)\right\rangle=\Delta_{\boldsymbol{\alpha}}\left(J(G)^{s}\right),
$$

so $\widetilde{H}_{m-2}\left(\Delta_{\boldsymbol{\beta}}\left(J\left(G^{\prime}\right)\right) ; k\right) \neq \mathbf{0}$. Together with Lemma 1.2 , this fact gives

$$
H_{\mathfrak{m}}^{m-1}\left(R / J\left(G^{\prime}\right)\right)_{\boldsymbol{\beta}} \neq \mathbf{0},
$$

whence depth $R / J\left(G^{\prime}\right) \leqslant m-1$. On the other hand, as $\nu_{0}\left(G^{\prime}\right) \leqslant m$, by Theorems 2.3 and 3.2 we imply that $\operatorname{depth} R / J\left(G^{\prime}\right) \geqslant m-1$. Hence, $\operatorname{depth} R / J\left(G^{\prime}\right)=m-1$, and hence $G^{\prime}$ consists of $m$ disjoint edges by Lemma 3.5.

From the structure of $G$ we imply that $G^{\prime}$ is just $m$ disjoint edges:

$$
\{1, m+1\},\{2, m+2\}, \ldots,\{m, 2 m\} \text {. }
$$


Therefore,

$$
\Delta_{\boldsymbol{\alpha}}\left(J(G)^{s}\right)=\left\langle F_{1, m+1}, F_{2, m+2}, \ldots, F_{m, 2 m}\right\rangle .
$$

For each $i=1, \ldots, m-1$, since $F_{i, m+i} \in \Delta_{\boldsymbol{\alpha}}\left(J(G)^{m}\right)$ and $F_{i, m+i+1} \notin \Delta_{\boldsymbol{\alpha}}\left(J(G)^{m}\right)$, by (15) we have $\alpha_{i}+\alpha_{m+i} \leqslant s-1$ and $\alpha_{i}+\alpha_{m+i+1} \geqslant s$, and hence $\alpha_{m+i}<\alpha_{m+i+1}$. It follows that

$$
\alpha_{m+1}<\alpha_{m+2}<\ldots<\alpha_{2 m} .
$$

In particular, $\alpha_{2 m} \geqslant m-1$. As $F_{m+1,2 m} \in \Delta_{\boldsymbol{\alpha}}\left(J(G)^{s}\right)$ we obtain

$$
s-1 \geqslant \alpha_{m+1}+\alpha_{2 m} \geqslant m-1 .
$$

Therefore $s \geqslant m$, as required.

Acknowledgement. We would like to thank the referee for many helpful comments. This work is partially supported by NAFOSTED (Vietnam) under the grant number 101.04-2015.02. The first author is also partially supported by Thai Nguyen university of Science under the grant number ĐH2016-TN06-03.

\section{References}

1. Bandari, S., Herzog, J. and Hibi, T., Monomial ideals whose depth function has any given number of strict local maxima, Ark. Mat. 52 (2014), 11-19.

2. Berge, C., Hypergraphs: Combinatorics of Finite Sets, North-Holland, New York, 1989.

3. Brodmann, M., The asymptotic nature of the analytic spread, Math. Proc. Cambridge Philos. Soc. 86 (1979), 35-39.

4. Brun, W. and Herzog, J., Cohen-Macaulay Rings, Cambridge Studies in Advanced Mathematics 39, Cambridge University Press, Cambridge, 1993.

5. Cowsik, R. C. and Nori, M. V., Fibers of blowing up, J. Indian Math. Soc. (N.S.) 40 (1976), 217-222.

6. Constantinescu, A., Pournaki, M. R., Seyed Fakhari, S. A., Terai, N. and YASSEmi, S., Cohen-Macaulayness and limit behavior of depth for powers of cover ideals, Comm. Algebra 43 (2015), 143-157.

7. Constantinescu, A. and Varbaro, M., Koszulness, Krull dimension, and other properties of graph-related algebras, J. Algebraic Combin. 34 (2011), 375-400.

8. Hà, H. T., Trung, N. V. and Trung, T. N., Depth and regularity of powers of sums of ideals, Math. Z. 282 (2016), 819-838.

9. Hà, H. T. and Sun, M., Squarefree monomial ideals that fail the persistence property and non-increasing depth, Acta Math. Vietnam. 40 (2015), 125-137.

10. Herzog, J. and Hibi, T., The depth of powers of an ideal, J. Algebra 291 (2005), $534-550$.

11. Herzog, J. and Hibi, T., Monomial Ideals, GTM 260, Springer, 2010. 
12. Herzog, J., Hibi, T. and Trung, N. V., Vertex cover algebras of unimodular hypergraphs, Proc. Amer. Math. Soc. 137 (2009), 409-414.

13. Herzog, J. and Qureshi, A. A., Persistence and stability properties of powers of ideals, J. Pure Appl. Algebra 219 (2015), 530-542.

14. Herzog, J. and Vladoiu, M., Squarefree monomial ideals with constant depth function, J. Pure Appl. Algebra 217 (2013), 1764-1772.

15. Hoa, L. T., Kimura, K., Terai, N. and Trung, T. N., Stability of depths of symbolic powers of Stanley-Reisner ideals, J. Algebra 473 (2017), 307-325.

16. HoA, L. T. and Trung, T. N., Partial Castelnuovo-Mumford regularities of sums and intersections of powers of monomial ideals, Math. Proc. Cambridge Philos. Soc. 149 (2010), 1-18.

17. Hochster, M., Cohen-Macaulay rings, combinatorics, and simplicial complexes, in Ring Theory II, Lect. Notes in Pure and Appl. Math. 26, pp. 171-223, M. Dekker, 1977.

18. Kaiser, T., Stehlík, M. and ŠKrekovski, R., Replication in critical graphs and the persistence of monomial ideals, J. Combin. Theory Ser. A 123 (2014), 239-251.

19. Miller, E. and Sturmfels, B., Combinatorial Commutative Algebra, Springer, 2005.

20. Minh, N. C. and Trung, N. V., Cohen-Macaulayness of powers of two-dimensional squarefree monomial ideals, J. Algebra 322 (2009), 4219-4227.

21. Morey, S., Depths of powers of the edge ideal of a tree, Comm. Algebra 38 (2010), 4042-4055.

22. Nam, L. D. and Vabaro, M., When depth is comming soon, J. Algebra 445 (2016), $181-192$.

23. SchriJver, A., Theory of Linear and Integer Programming, John Wiley \& Sons, 1998.

24. TAKayama, Y., Combinatorial characterizations of generalized Cohen-Macaulay monomial ideals, Bull. Math. Soc. Sci. Math. Roumanie (N.S.) 48 (2005), 327-344.

25. Trung, T. N., Stability of depths of powers of edge ideals, J. Algebra 452 (2016), $157-187$.

Nguyen Thu Hang

Thai Nguyen College of Sciences

Thai Nguyen University

Thai Nguyen

Vietnam

nguyenthuhang0508@gmail.com

\author{
Tran Nam Trung \\ Institute of Mathematics \\ VAST \\ 18 Hoang Quoc Viet \\ Hanoi \\ VietNam \\ tntrung@math.ac.vn
}

